\title{
Design of Strongly Modulating Pulses to Implement Precise Effective Hamiltonians for Quantum Information Processing
}

\author{
Evan M. Fortunato ${ }^{\dagger}$, Marco A. Pravia ${ }^{\dagger}$, Nicolas Boulant ${ }^{\dagger}$, \\ Grum Teklemariam $^{\ddagger}$, Timothy F. Havel ${ }^{\dagger}$, David G. Cory ${ }^{\dagger}$ \\ ${ }^{\dagger}$ Department of Nuclear Engineering, Massachusetts Institute of Technology, Cambridge, MA 02139 \\ ${ }^{\ddagger}$ Department of Physics, Massachusetts Institute of Technology, Cambridge, MA 02139
}

\begin{abstract}
We describe a method for improving coherent control through the use of detailed knowledge of the system's Hamiltonian. Precise unitary transformations were obtained by strongly modulating the system's dynamics to average out unwanted evolution. With the aid of numerical search methods, pulsed irradiation schemes are obtained that perform accurate, arbitrary, selective gates on multi-qubit systems. Compared to low power selective pulses, which cannot average out all unwanted evolution, these pulses are substantially shorter in time, thereby reducing the effects of relaxation. Liquid-state NMR techniques on homonuclear spin systems are used to demonstrate the accuracy of these gates both in simulation and experiment. Simulations of the coherent evolution of a 3-qubit system show that the control sequences faithfully implement the unitary operations, typically yielding gate fidelities on the order of 0.999 and, for some sequences, up to 0.9997 . The experimentally determined density matrices resulting from the application of different control sequences on a 3-spin system have overlaps of up to 0.99 with the expected states, confirming the quality of the experimental implementation.
\end{abstract}

\section{INTRODUCTION}

The past decade has seen a substantial interest in improving coherent quantum control. Coherent control had origins in both nuclear magnetic resonance (NMR) [1, 2] and optical spectroscopy [3]. For an overview of advances in both fields, the reader is referred to [5]. Since coherent control's inception, many different techniques have been used both to improve selectivity and to reduce the duration of control pulses. For spin systems, the Fourier transform has been used to approximate the excitation profile in the limit of low power and no spin-spin couplings [6] and more complete analytic solutions have been developed to aid in general pulse design and analysis [7 12]. Alternatively, very sophisticated shaped pulses have been designed using a variety of computer-aided methods [13,14] or feedback from system observables [15]. Equivalent analytic theories [16,17], computer-aided methods [18] and feedback techniques [19,20] have also been developed by the optics community. Similar techniques are also used in other fields such as the control of trapped ions [21,22].

The development of liquid-state NMR systems as prototype quantum information processors [23,24] has enabled experimental demonstration of quantum algorithms [25 27], quantum error correction [28 29], and quantum simulations [30]. These experiments built upon well-established spectroscopic techniques developed over the past four decades, such as using low-power (soft) shaped radio-frequency (RF) pulses to obtain selective operations. However, the selective pulses employed to date have the disadvantage that low power implies long duration. This not only introduces errors due to relaxation, or decoherence, but also allows significant evolution under the action of the internal Hamiltonian. In the past, this evolution was rarely of concern because there was little importance placed on implementing a particular operation. For example, in spectroscopy there are entire classes of propagators that selectively excite a single spin from its equilibrium state, but for applications such as quantum computing the transformation must act as expected for all input states. A second problem with soft-pulse techniques is that selective pulses simultaneously applied to different spins interfere with each other, thus causing significant deviation from the desired action [31]. To address some of these problems, several groups precalculate these errors and incorporate corrections into their analysis and pulse design [32, 33]. However, not all errors can be corrected using these techniques [34], and it would be preferable to average out unwanted evolution by the use of strong control fields, so that no additional corrections are required.

In this paper, we present a procedure for finding highpower pulses that strongly modulate the system's dynamics to produce precisely a desired spin-selective unitary propagator. These operations, or gates, allow arbitrary rotations of each spin around independent single-spin axes, while refocusing the internal evolution. They are "self-contained," in the sense that they can be placed back to back in longer sequences without requiring additional computational resources or postexperiment corrections. By using high-power, pulse durations are decreased by almost an order of magnitude, thereby significantly reducing the effects of relaxation. Finally, the use of strong modulation also opens the possibility of incorporating robustness against slowly varying or time independent incoherent errors such as those caused by static field or RF inhomogeneities [35-37]. Our control methods are the first to combine all of these features.

The pulses presented here have been applied in recent Quantum Information Processing (QIP) experiments to demonstrate algorithms [27], study notions of measurement [38], and test new methods for noise control [39]. They promise to be increasingly useful in future NMR QIP experiments, where larger numbers of qubits will necessitate increasing the number of homonuclear spins. In addition, these methods can be adapted to develop improved pulses for selective spectroscopy [40] and imaging [41]. Finally, although presented within the context of NMR, these methods are applicable to any system where the total Hamiltonian is well known and the external degrees of freedom allow for universal control, both requirements of any quantum information pro- 
cessor.

\section{CONTROL OF QUANTUM INFORMATION}

In the standard model of quantum computing, an algorithm can be expressed as a series of unitary operations that maps a set of input states to a particular set of output states. The physical implementation of an algorithm requires the use of a quantum system with a Hamiltonian that contains a sufficient set of externally controlled parameters to allow for the generation of a universal set of gates [42]. The task of control is to find a time-dependent sequence of values for these control parameters that modulates the system's dynamics in order to generate a particular gate to the required precision.

Given a control sequence, solving for the effective Hamiltonian is straightforward. Unfortunately, going the other way is much more difficult. That is, finding an RF waveform that produces a propagator with desired properties is an inverse problem. Traditionally, analytic techniques, such as average Hamiltonian theory [2], have been used to determine an appropriate control sequence. With modern computer resources, numerical methods provide a more efficient and accurate solution to this problem.

\section{A. Gate Fidelity as a Metric for Control}

A metric of a gate's performance should describe the quality of a general transformation, including the possibility of non-unitary evolution. Unfortunately, such information is not conveniently accessible by experiment, so we choose a metric comprised only of sets of state measurements. For an input state, $\rho_{i n}$, the ideal transformation maps the system to a theoretical output state, $\rho_{t h}$, i.e.,

$$
\rho_{\text {in }} \longrightarrow \rho_{t h}
$$

On the other hand, a simulated or experimentally implemented control sequence will produce a different output state, $\rho_{\text {out }}$, i.e.,

$$
\rho_{\text {in }} \longrightarrow \rho_{\text {out }} \text {. }
$$

Noting that $\rho$ is Hermitian, the projection between these two states, defined as

$$
P\left(\rho_{\text {th }}, \rho_{\text {out }}\right)=\frac{\operatorname{trace}\left(\rho_{\text {th }} \rho_{\text {out }}\right)}{\sqrt{\operatorname{trace}\left(\rho_{\text {th }}^{2}\right) \operatorname{trace}\left(\rho_{\text {out }}^{2}\right)}},
$$

quantifies how similar in 'direction' the two states are. This metric is analogous to the dot product between two vectors, varying from -1 for anti-parallel states to 1 for identical states. A value of zero indicates orthogonal density matrices. In order to account for non-unitary evolution, a second term multiplies the projection yielding the attenuated correlation, namely,

$$
\begin{aligned}
C\left(\rho_{\text {th }}, \rho_{\text {out }}\right)= & P\left(\rho_{\text {th }}, \rho_{\text {out }}\right) \sqrt{\frac{\operatorname{trace}\left(\rho_{\text {out }}^{2}\right)}{\operatorname{trace}\left(\rho_{\text {in }}^{2}\right)}} \\
& =\frac{\operatorname{trace}\left(\rho_{\text {th }} \rho_{\text {out }}\right)}{\sqrt{\operatorname{trace}\left(\rho_{\text {th }}^{2}\right) \operatorname{trace}\left(\rho_{\text {in }}^{2}\right)}} .
\end{aligned}
$$

We define the gate fidelity, F, of a transformation as

$$
F=\overline{C\left(\rho_{\text {th }}, \rho_{\text {out }}\right)}
$$

where $\bar{C}$ represents the average attenuated correlation over an orthonormal set of input density operators (i.e., Trace $\left[\rho_{j} \rho_{k}\right]=\delta_{j k}$ ) that span the Hilbert space. It should be noted that $F$ is maximized (with a value of one) when the implemented and ideal transformations are the same, and is insensitive to differences in the global phase between the ideal and implemented transformation.

We can derive a useful alternate form for the gate fidelity in terms of the actual and theoretical transformations instead of the input-output state relations. This form is both easier to compute and has intuitive appeal in that knowledge of the transformation can be directly translated to gate fidelities. First we assume that our ideal transformation is unitary, and the implemented transformation is a completely positive, trace-preserving linear map [43]. In other words, the implemented transformation takes normalized density operators to normalized density operators and if the system starts as subsystem of an entangled system, then the full system's density operator also maps in a reasonable way. Under these assumptions, Eq. (1) and (2) are explicitly given by

$$
\rho_{t h}=U_{t h} \rho_{i n} U_{t h}^{\dagger},
$$

and

$$
\rho_{\text {out }}=\sum_{\mu} A_{\mu} \rho_{i n} A_{\mu}^{\dagger}
$$

where the $A_{\mu}$ satisfy

$$
\sum_{\mu} A_{\mu}^{\dagger} A_{\mu}=\mathbb{1}
$$

We now show that the gate fidelity reduces to

$$
F=\sum_{\mu}\left|\operatorname{Trace}\left(U_{t h}^{\dagger} A_{\mu}\right) / N\right|^{2},
$$

where $\mathrm{N}$ is the dimension of the Hilbert space. Using the normalized Pauli basis, $\sigma_{j}$, as the orthonormal input density operators and the cyclic properties of the trace, Eq. (6) becomes

$$
\begin{aligned}
F= & \sum_{j=1}^{N^{2}} \operatorname{Tr}\left[\left(U_{t h} \sigma_{j} U_{t h}^{\dagger}\right)\left(\sum_{\mu} A_{\mu} \sigma_{j} A_{\mu}^{\dagger}\right)\right] / N^{2} \\
& =\sum_{j=1}^{N^{2}} \operatorname{Tr}\left[\sigma_{j} \sum_{\mu} U_{t h}^{\dagger} A_{\mu} \sigma_{j} A_{\mu}^{\dagger} U_{t h}\right] / N^{2} .
\end{aligned}
$$

Expanding the product of $U_{t h}^{\dagger} A_{\mu}$ in terms of the orthonormal Pauli basis $\left(U_{t h}^{\dagger} A_{\mu}=\sum_{k} B_{\mu}^{k} \sigma_{k}\right)$, yields 


$$
\begin{aligned}
&=\sum_{j \mu} \operatorname{Tr} {\left[\sigma_{j}\left(\sum_{k} B_{\mu}^{k} \sigma_{k}\right) \sigma_{j}\left(\sum_{m} B_{\mu}^{m *} \sigma_{m}\right)\right] / N^{2} } \\
&=\sum_{j \mu k m} B_{\mu}^{k} B_{\mu}^{m *} \operatorname{Tr}\left[\sigma_{j} \sigma_{k} \sigma_{j} \sigma_{m}\right] / N^{2} .
\end{aligned}
$$

Because the $\sigma$ basis is orthogonal, only terms where $k=m$ contribute. Therefore, Eq. (14) reduces to

$$
F=\sum_{j \mu k}\left|B_{\mu}^{k}\right|^{2} \operatorname{Tr}\left[\sigma_{j} \sigma_{k} \sigma_{j} \sigma_{k}\right] / N^{2} .
$$

If $\sigma_{k}$ is not proportional to identity, it will anti-commute with exactly half the $\sigma_{j}$ terms in the sum, while commuting with the other half. Therefore, two sets of terms cancel and have no contribution to F. Defining $\sigma_{1}$ to be the element that is proportional to identity, Eq. 15) further reduces to

$$
\begin{gathered}
F=\sum_{j \mu}\left|B_{\mu}^{1}\right|^{2} \operatorname{Tr}\left[\sigma_{j} \sigma_{j}\right] / N^{3} \\
=\sum_{j \mu}\left|B_{\mu}^{1}\right|^{2} / N^{3}=\sum_{\mu}\left|B_{\mu}^{1}\right|^{2} / N .
\end{gathered}
$$

This is clearly equal to Eq. 10. Thus, the gate fidelity corresponds to how well the actual transformation reverses the action of $U_{t h}^{\dagger}$. In this form it is obvious that the gate Fidelity is independent of which orthonormal basis of input states are used as $\rho_{i n}$.

\section{B. NMR as an Example System}

As an example, liquid-state NMR is used to demonstrate how to find control sequences to implement particular gates. In NMR, spins in a large static magnetic field (in our case, 9.7 T) are controlled via external RF pulses. The internal spin Hamiltonian is composed of both Zeeman interactions with the applied field modified by electron screening (chemical shift) and scalar couplings with other spins. Together these provide the QIP requirements of addressability and conditional logic respectively. In terms of spin operators, the internal Hamiltonian is

$$
H_{\text {int }}=\sum_{k=1}^{n} \omega_{k} I_{z}^{k}+2 \pi \sum_{j>k}^{n} \sum_{k=1}^{n} J_{k j} I^{k} \cdot I^{j},
$$

where $\omega_{k}$ represent the chemical shifts of the spins, $J_{k j}$ the coupling constant between spins $k$ and $j$, and $n$ is the number of spins. The external Hamiltonian describing the coupling between the spins and an oscillating RF field generated by a single transmitter is

$H_{e x t}\left(\omega_{R F}, \phi, \omega, t\right)=\sum_{k=1}^{n} e^{-i\left(\omega_{R F} t+\phi\right) I_{z}^{k}}\left(-\omega I_{x}^{k}\right) e^{i\left(\omega_{R F} t+\phi\right) I_{z}^{k}}$,

where $\omega_{R F}$ is the transmitter's angular frequency, $\phi$ the initial phase, and $\omega$ the power [44]. Of course, additional species can be added by including appropriate terms in $H_{\text {int }}$ and an additional $H_{\text {ext }}$ for each additional RF field.

Using this knowledge of the internal Hamiltonian and the form of the external Hamiltonian, the parameter values that generate the desired gate must be determined. Here, a quality factor $Q=1-\sqrt{F}$ is minimized by searching through the mathematical parameter space using the Nelder-Mead Simplex algorithm [45]. While this function has many local minima, the Simplex algorithm often succeeds in finding satisfactory solutions. Our goal is to show that sufficient, implementable control sequences can be found. Finding the optimal solution is much more challenging and based on our system and control parameter values, is not expected to improve pulse performance significantly. We have parameterized the control sequence as a cascade of RF pulses with fixed power, transmitter frequency, initial phase, and pulse duration $(\tau)$. As will be seen, this is a particularly convenient and completely general parameterization, but we make no claims that it is the only, nor necessarily the best choice. If the RF power is constant over the duration of a pulse, i.e., the pulse's amplitude is square, the total Hamiltonian $H_{t o t}=H_{i n t}+H_{\text {ext }}$ can be made time independent by transforming into the frame that rotates at the frequency of the transmitter. This allows the Liouville-von Neumann equation of motion to be solved by a single diagonalization. Initially, the starting density matrix is the same in both frames $(\tilde{\rho}(0)=\rho(0))$, so that at the end of the pulse, the density matrix in the new frame is given by

$$
\tilde{\rho}(\tau)=e^{-i H_{e f f} \tau} \rho(0) e^{i H_{e f f} \tau},
$$

where $H_{\text {eff }}$ is the effective Hamiltonian in the new frame [46]. Transforming this density matrix back to the original rotating frame gives

$$
\rho=U_{z}(\tau)^{-1} e^{-i H_{e f f} \tau} \rho(0) e^{i H_{e f f} \tau} U_{z}(\tau),
$$

where

$$
U_{z}(\tau)=\left(e^{i \omega_{R F} \sum_{k=1}^{n} I_{z}^{k} \tau}\right) .
$$

Therefore, in the original rotating frame, the transformation is given by

$$
U_{\text {period }}(\tau)=U_{z}^{-1}(\tau) e^{-i H_{e f f} \tau} .
$$

Because the evolution under the whole sequence is given in the original rotating frame, no additional resources are required to concatenate pulses, nor is any mathematical correction required at the end of an experiment.

Cascading these periods yields the net transformation

$$
U_{n e t}=\prod_{m=1}^{N} U_{z}^{-1}\left(\tau_{m}\right) e^{-i H_{e f f}^{m}\left(\omega^{m}, \omega_{R F}^{m}, \phi^{m}\right) \tau_{m}}
$$

where the index $m$ refers to the $m^{\text {th }}$ period, i.e., to the $m^{\text {th }}$ square pulse, with a corresponding set of 4 parameters. In other words, $N$ constant amplitude pulse periods, each with a different transmitter frequency and initial phase, are applied in series. Clearly, a single period is not sufficient to generate 
an arbitrary transformation; therefore the number of periods is increased until a suitable net transformation is found. Using desktop computing resources, this yields convergence times for three- and four-spin systems that are typically seconds to minutes.

In addition to the desired propagator, $U_{\text {ideal }}$, an initial set of starting parameters for the pulse shape is required. While this initial guess must be reasonable (i.e., in the vicinity of the solution), many different starting points typically converge to equally deep minima. We have observed that the number of acceptable solutions for this parameterization is very large, allowing experimental implementation issues to be considered. For example, experimental limitations do not allow arbitrarily high powers or frequencies to be implemented. To keep the algorithm from returning infeasible solutions, a penalty function that increases as the parameter value moves towards infeasible solutions is added to the quality factor. Penalty functions are also used to guide the algorithm towards more favorable pulse solutions. In our case, penalties are placed on high powers, large frequencies, and negative- or long-time periods.

\section{SIMULATIONS}

The methods described above were used to obtain a set of pulses that implement each of a set of important single-spin gates. To study the performance of these gates, propagators for each of the pulses were simulated under different conditions. First, the performance of the pulses under idealized experimental conditions is considered. Second, the gate fidelity is simulated as a function of systematic distortions of the pulse parameters. From these results, the relative importance of implementation precision is determined. Finally, simulations show that a pulse generates quite different evolutions as a test spin's resonant frequency is varied over a range of chemical shifts.

\section{A. Ideal Pulse Simulations}

Pulses were created for three- $\left({ }^{13} C\right.$-labeled Alanine $)$ and four- $\left({ }^{13} \mathrm{C}\right.$-labeled Crotonic acid) spin homonuclear systems. The chemical shifts and scalar coupling constants for each of these systems are listed in Fig. 1. As a representative set, each of the single spin $\pi / 2$, and nearest-neighbor paired $\pi$ pulses were simulated with the relevant characteristics summarized in Table If and example waveforms shown in Fig. 2. The duration of the pulses are on the order of $200 \mu$ s for the three-spin system and $420 \mu$ s for the four-spin system, both significantly shorter than those that could be obtained using low-power pulses. The average fidelities for each system are 0.9995 and 0.995 , demonstrating that, at least under ideal conditions, control sequences that implement the desired transformation with high fidelity can be found.

The ultimate goal of control in quantum computing is to attain fault-tolerant computation. While it has been proven that perfect control is not required [47], estimates of the precision needed vary from 0.9999 to 0.999999 depending on the assumptions used. These simulations predict an achievable level of control that approaches the most optimistic estimates for fault tolerant computation. As expected, the pulse duration decreases with increasing chemical shifts dispersion (selectivity condition) and, for the case that $J_{j k}<<\left|\omega_{k}-\omega_{j}\right|$, the fidelity of the sequence decreases with increasing ratio of the couplings (bilinear terms) to chemical shift.

\section{B. Variations in the External Hamiltonian}

The external RF parameters are determined by a minimization procedure, suggesting that small variations of the external parameters should have little effect on the quality of the pulses. To check this assumption, the gate fidelity was calculated as each of the six pairs of the four control parameters were varied over a range of errors typical of an experimental implementation. As a sample set, one pulse for each of the two systems is presented here. The results shown in Fig. 3 demonstrate the natural robustness against typical variations in the initial phase, frequency, and duration of each period. Clearly, the sequence is most sensitive to power variations. For the pulses listed in Table If, if the power's amplitude is changed by $5 \%$ the average fidelity falls to $0.96 \pm 0.01$ for Alanine pulses and $0.94 \pm 0.04$ for Crotonic acid pulses. For the 25 pulses used in [39] the average fidelity at $5 \%$ amplitude deviation is $0.97 \pm 0.02$. For $10 \%$ deviation, the gate fidelity drops to $0.86 \pm 0.03$ for the Alanine pulses and $0.81 \pm 0.12$ for the Crotonic acid pulses. This pulse sensitivity to RF amplitude suggests that RF inhomogeneity may be a leading cause of experimental errors. While techniques to select homogeneous regions are available [33,48], the loss in signal to noise is significant, especially if multiple coils are used. Instead, because these errors are incoherent in nature, it is possible to design pulse sequences that refocus such homogeneities. Preliminary results confirm that sequences can be re-optimized in the presence of significant inhomogeneity.

\section{Variations in the Internal Hamiltonian}

For NMR spectroscopy, the goal is to excite selectively spins in a band of frequencies leaving all other possible spins (with unknown precession frequencies) along the $z$ axis. This requires that the propagator for spins at any other frequency be, at most a phase change. With detailed knowledge of the internal Hamiltonian, the effect of the applied RF field needs only be considered at the resonance frequencies of the chemical species present in the given molecule. By relaxing the requirement that the effective Hamiltonian be zero for all chemical shifts other than those in the band of excitation, an RF shape can be found that more efficiently implements the desired gate for the frequencies of concern yielding high-power yet selective pulses. To demonstrate this idea more clearly, the gate fidelities of the two sample pulses considered in the sub-section B were calculated as a function of a test spin's 
resonant frequency. As can be seen in Fig. 4 , the fidelity is close to unity only near the resonance frequency for which the pulse was designed to work. This stresses the necessity of having accurate knowledge of the system's Hamiltonian. On the other hand, looking at the region immediately around the resonance we see the fidelity falls off quite slowly. This implies that small variations in the chemical shift do not significantly affect the fidelity of the pulses. For example, in the experiments presented below, the unwanted scalar couplings to the hydrogen atoms, which are equivalent to errors in the resonance frequency, were automatically refocused by the control pulse. It should be noted that no constraint was used to require this robustness, but that it results from the use of strongly modulating pulses. If this natural robustness is not sufficient, additional constraints can be added. Of course, this robustness also implies that selective pulses will be harder to design for spin systems where the spectrum is dense.

\section{EXPERIMENTAL DEMONSTRATIONS}

In this section we experimentally demonstrate the efficacy of a representative set of pulses via both spectra and reconstructed density matrices. Each waveform was discretized by sampling at a constant rate that was faster than the largest phase modulation during any of the periods (sample times of order $0.5 \mu \mathrm{s}$ ). Simulations of the sampled waveform confirmed that the decrease in the gate fidelity was acceptably small. All experimental tests were carried out on the three carbons of ${ }^{13} \mathrm{C}$-labeled Alanine (see Fig. 1 for internal Hamiltonian parameters) using a Bruker Avance spectrometer.

First, a series of selective single-spin pulses were applied to the thermal state to demonstrate that these selective rotations fully refocus the internal Hamiltonian and so can be concatenated arbitrarily. Fig. 5 shows sample sequences and the resulting spectra. In addition, because the internal Hamiltonian is fully refocused, applying selective transformations on different spins sequentially has an effect equivalent to applying all the appropriate transformations to each of the spins simultaneously (neglecting relaxation). With simultaneous, fully self-refocused $\pi$ pulses, selective couplings can be efficiently implemented using previously published techniques [46, 49, and is demonstrated in Fig. 5.

Second, the projection and attenuated correlation between the expected and experimentally determined results of different control sequences were measured for a set of input states. While spectra contain information about the observables of the current state of the system (single-spin transitions in the case of NMR), a single spectrum does not contain enough information to reconstruct the entire state of the system (density matrix). By using "read-out" pulses that rotate different elements of the density matrix into the single-spin transitions, every term of the density matrix can be determined [50]. In our case, seven repetitions of the experiment, each with a different readout pulse, are sufficient to reconstruct the density matrix [51]. Using this method to determine $\rho_{\text {out }}$, the projections and attenuated correlations, averaged over three different input states, and under the action of six different pulses, are measured and listed in Table III. The input states considered are of the form

$$
\rho_{\text {in }}=I_{j}^{1}+I_{j}^{2}+I_{j}^{3}
$$

for $j=x, y, z$.

Because the pulses are short with respect to the decoherence times, which are all greater than $200 \mathrm{~ms}$, the difference between the projection and the correlation indicates incoherent evolution (e.g., RF inhomogeneity) has caused significant errors. This is demonstrated by the fact that the ratio of the correlation to the projection, or the attenuation, is on the order of the projection (see Table III). This further supports the need to address errors caused by inhomogeneous effects. Robustness to inhomogeneity can be added as a criterion in pulse determination; the additional resource requirements (time, power, etc.), however, are not known. Decreases in the coherent errors are also desirable, but such improvements must first be sought through improved experimental implementation. While the goal of fault tolerance clearly hasn't been met, these results indicate that experimental implementation is nearing ideal simulation results.

\section{CONCLUSIONS}

The ability to implement faithfully a desired unitary transformation is at the heart of any future implementation of a quantum computer. Any control technique should minimize the effects of decoherent errors while retaining the required addressability. We have demonstrated a method to find control sequences that use detailed knowledge of the system's Hamiltonian and high-power pulses to create a desired gate by strongly modulating the system's dynamics. These gates are short in duration yet selective, implementing the correct unitary transformation. The effect of these control sequences has been simulated under various conditions and experimentally demonstrated by NMR. Finally, avenues for future improvements are within the reach of current technologies.

\section{ACKNOWLEDGEMENTS}

We are grateful to L. Viola, E. Knill and R. Laflamme for stimulating discussions. This work was supported by the National Security Agency and Advanced Research and Development Activity under Army Research Office contract number DAAD19-01-1-0519, by the Defense Sciences Office of the DARPA under contract number MDA972-01-1-0003, and by NSF. 
[1] I.I. Rabi, S. Millman, P. Kusch, and J.R. Zacharias, Phys. Rev. 55526 (1939).

[2] U. Haeberlen, and J.S. Waugh, Phys. Rev. 175, 453-67 (1968).

[3] A.F. Haught, Annu. Rev. Phys. Chem. 19, 343 (1968).

[4] W.S. Warren, H. Rabitz, and M. Dahleh, Science 259, 15811589 (1993).

[5] W.S. Warren, Science 242, 878-84 (1988).

[6] A. Sodickson, D. G. Cory, Prog. Nucl. Magn. Res. Spectrosc. 33, 77 (1998).

[7] C. Counsell, M.H. Levitt, and R.R. Ernst, J. Magn. Reson. 63, 133-41 (1985).

[8] S. Connolly, D. Nishimura, and A. Macovski, IEEE Transactions on Medical Imaging MI-5, 106-15 (1986).

[9] R. Tycko, E. Schneider, and A. Pines, J. Chem. Phys. 81, 680688 (1984)

[10] P.G. Morris, D.E. Rourke, D.J.O. McIntyre, and A. Al-Beshr Magnetic Resonance Materials in Physics, Biology, \& Medicine 2, 279-83 (1994).

[11] J. Stoustrup, O. Schedletzky, S.J. Glasser, C. Griesinger, N.C. Nielsen, and O.W. Sørensen, Phys. Rev. Lett. 74 2921-4 (1995).

[12] L. Emsley, and G. Bodenhausen, J. Magn. Reson. 97, 135-48 (1992).

[13] H. Green, and R. Freeman, J. Magn. Reson. 93, 93-141 (1991).

[14] B. Ewing, S.J. Glasser, and G.P. Drobny, J. Magn. Reson. 98 381-7 (1992).

[15] J.L. Schiano, A.G. Webb, and R.L. Magin, IEEE Trans. on Med. Imag. 11, 203-14 (1992).

[16] A.P. Peirce, M. Dahleh, and H. Rabitz, Phys. Rev. A 374950 (1988).

[17] W.S. Warren and A.H. Zewail, J. Chem. Phys. 78 2279-97 (1983).

[18] M. Dahleh, A.P. Peirce, and H. Rabitz, Phys. Rev. A 42, 1065-79 (1990).

[19] A. Assion, T. Baumert, M. Bergt, T. Brixner, B. Kiefer, V. Seyfried, M. Strehle, and G. Gerber, Science 282, 919 (1998)

[20] B.J. Pearson, J.L. White, T.C. Weinacht, and P.H. Bucksbaum, Phys. Rev. A 63063412 (2001)

[21] A. Sørensen and K. Molmer, Fortschritte der Physik-Progress of Physics 48 811-21 (2000).

[22] D. Kielpinski, V. Meyer, M.A. Rowe, C.A. Sackett, W.M. Itno, C. Monroe, and D.J. Wineland, Science 291 1013-1015 (2001).

[23] D.G. Cory, A.F. Fahmy and T.F. Havel, Proc. Natl. Acad. Sci. 94, 1634-1639 (1997).

[24] N.A. Gershenfeld and I.L. Chuang, Science 275, 350-356 (1997).

[25] J.A. Jones, and M. Mosca, J. Chem. Phys. 109, 1648-1653 (1998)

[26] I.L. Chuang, N. Gershenfeld, M. Kubinec, and D. Leung, Phys. Rev. Lett. 80, 3408-11 (1998).

[27] Y.S. Weinstein, M.A. Pravia, E.M. Fortunato, S. Lloyd, and D.G. Cory, Phys. Rev. Lett. 86 1889-91 (2001).

[28] D. G. Cory, W. Maas, M. Price, E. Knill, R. Laflamme, W. H. Zurek, T. F. Havel and S. S. Somaroo, Phs. Rev. Lett. 81, 21522155 (1998)

[29] E. Knill, R. Laflamme, R. Martinez, and C. Negrevergne, Phys.
Rev. Lett. 86, 5811 (2001).

[30] S. Somaroo, C.-H. Tseng, T.F. Havel, R. Laflamme, and D.G. Cory, Phys. Rev. Lett. 82, 5381-84 (1999).

[31] F. Bloch and A. Siegert, Phys. Rev. 57, 522-527 (1940).

[32] M. Steffen, L.M.K Vandersypen, and I.L. Chuang, J. Magn. Reson. 146, 369-74 (2000).

[33] E. Knill, R. Laflamme, R. Martinez and C-H. Tseng, Nature 404, 368-70 (2000).

[34] For instance, not all errors can be represented as a composition of phase shifts, $\sigma_{z} \sigma_{z}$ couplings and ideal $\pi / 2$ or $\pi$ pulses.

[35] E.L. Hahn, Phys Rev. 9080, 580-594 (1950).

[36] H.Y. Carr, and E.M. Purcell, Phys. Rev. 94, 630-638 (1954).

[37] S. Meiboom, and D. Gill, Rev. Sci. Instrum. 29, 688-691 (1958).

[38] G. Teklemariam, E.M. Fortunato, M.A. Pravia, T.F. Havel and D.G. Cory, Phys. Rev. Lett. 86, 5845-49 (2001).

[39] L. Viola, E.M. Fortunato, M.A. Pravia, E. Knill, R. Laflamme, and D.G. Cory, Science 2932059 (2001).

[40] J. Huth, N.D. Kurur, and G. Bodenhausen, J. Magn. Reson. 118, 286-90 (1996).

[41] R.E. Gordon, P.E. Hanley, D. Shaw, D.G. Gadian, G.K. Radda, P. Styles, P.J. Bore, and L. Chan, Nature 287736 (1980).

[42] S. Lloyd, Phys. Rev. Lett. 75, 346-9 (1995).

[43] B. Schumacher, Phys. Rev. A 54, 2614-28 (1996).

[44] Actually, $\omega$ equals a spin's nutation rate caused by an RF field. Because this parameter is experimentally controlled by attenuating the RF power, it is commonly referred to as the pulse power.

[45] J.A. Nelder and R. Mead, Comput. J. 7, 308-13 (1965).

[46] R.R. Ernst, G. Bodenhausen, and A. Wokaun, Principles of $\mathrm{Nu}$ clear Magnetic Resonance in One and Two Dimensions. Oxford University Press, Oxford (1994).

[47] E. Knill, R. Laflamme, and W.H. Zurek, Science 279, 342-5 (1998).

[48] D.G. Cory, J. Magn. Reson. 103, 23-6 (1993).

[49] J.A. Jones, and E. Knill, J. Magn. Reson. 141, 322-5 (1999).

[50] I.L. Chuang, N. Gershenfeld, M. Kubinec, and D. Leung, Proc. R. Soc. London A 454, 447 (1998).

[51] The seven readout pulses used for density matrix reconstruction are: $\left.\left.\left.\left.\left.\pi / 2]_{y}^{1}, \pi / 2\right]_{y}^{3}, \pi / 2\right]_{x}^{3}, \pi / 2\right]_{y}^{1,2}, \pi / 2\right]_{x}^{2,3}, \pi / 2\right]_{y}^{1,2,3}$, $\pi / 2]_{x}^{1,2,3}$. 
TABLE I. Summary of the relevant characteristics for an example set of transformations. The three columns list the pulse duration (in $\mu s$ ), maximum power (in $\mathrm{kHz}$ ), and the gate fidelity of the simulated pulse. While the maximum power is relatively large, all powers are experimentally feasible. The pulses designed for the Crotonic acid sample require longer times and yield lower fidelities due to the decreased chemical separation and increase of coupling strengths.

\begin{tabular}{cccc}
\hline \hline Pulse & Pulse Time $(\mu s)$ & Max. Power $(k H z)$ & Fidelity \\
\hline$\left.\frac{\pi}{2}\right]_{x}^{1}$ & 202 & 7.9 & \\
$\left.\frac{\pi}{2}\right]_{x}^{2}$ & 221 & 9.3 & 0.9995 \\
$\left.\frac{\pi}{2}\right]_{x}^{3}$ & 212 & 9.0 & 0.9995 \\
$\left.\frac{\pi}{2}\right]_{x}^{12}$ & 194 & 8.5 & 0.9995 \\
$\left.\frac{\pi}{2}\right]_{x}^{23}$ & 179 & 9.2 & 0.9995 \\
$\left.\frac{\pi}{2}\right]_{x}^{123}$ & 163 & 10.3 & 0.9995 \\
$\pi]_{x}^{12}$ & 252 & 8.0 & 0.9995 \\
$\pi]_{x}^{23}$ & 129 & 10.3 & 0.9996 \\
\hline & Crotonic Acid Pulses & 0.9997 \\
$\left.\frac{\pi}{2}\right]_{x}^{1}$ & 389 & 8.5 & \\
$\left.\frac{\pi}{2}\right]_{x}^{2}$ & 610 & 4.8 & 0.9930 \\
$\left.\frac{\pi}{2}\right]_{x}^{3}$ & 392 & 6.3 & 0.9957 \\
$\left.\frac{\pi}{2}\right]_{x}^{4}$ & 559 & 7.6 & 0.9950 \\
$\pi]_{x}^{12}$ & 326 & 9.0 & 0.9923 \\
$\pi]_{x}^{23}$ & 315 & 11.3 & 0.9963 \\
$\pi]_{x}^{34}$ & 345 & 8.4 & 0.9932 \\
\hline \hline & & & 0.9962 \\
\hline
\end{tabular}


TABLE II. Summary of experimental data for the Alanine sample. For each of six $\pi / 2$ pulses, the experimentally determined density matrix is compared with the expected result. The first column (No Pulse) confirms that the experimental and expected inputs have almost unit overlap. Each of the other headings denote which spins are rotated by $\pi / 2$. In each case, the projection and attenuated correlation for each pulse is averaged over the three inputs $I_{j}^{1}+I_{j}^{2}+I_{j}^{3}$, for $j=x, y, z$. Because all pulses are short in comparison to the natural decoherence times, the attenuation gives an indication of the relative significance of the coherent and incoherent errors. Statistical uncertainties arising from errors in the tomographic density matrix reconstruction are order $1 \%$.

\begin{tabular}{lccccccc}
\hline \hline & No Pulse & Carbon 1 & Carbon 2 & Carbon 3 & Carbons 1\&2 & Carbons 2\&3 & All Carbons \\
\hline Projection & 0.996 & 0.992 & 0.995 & 0.996 & 0.993 & 0.996 & 0.992 \\
Correlation & NA & 0.977 & 0.985 & 0.986 & 0.975 & 0.984 & 0.986 \\
Attenuation & NA & 0.985 & 0.990 & 0.990 & 0.982 & 0.988 & 0.994 \\
\hline \hline
\end{tabular}




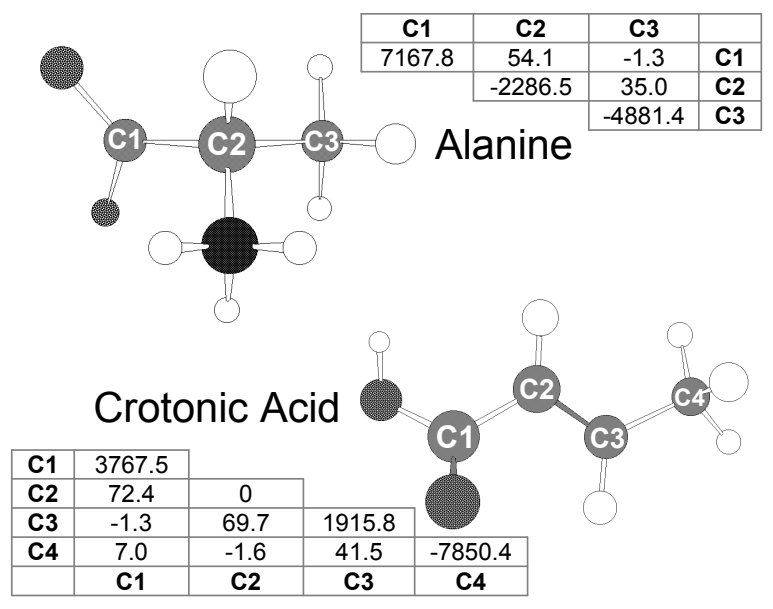

FIG. 1. Molecular structure and Hamiltonian parameters for both Alanine and Crotonic acid. The chemical shift of each of the carbon nuclei is given by the corresponding diagonal elements while the coupling strengths are given by the each of the different off-diagonal elements.

a) Three-spin $\pi / 2]_{x}^{3}$ pulse

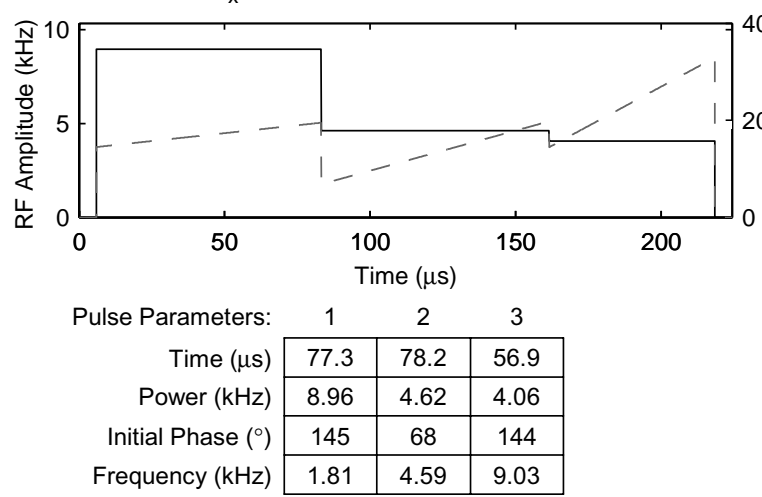

b) Four-spin $\pi]_{x}^{12}$ pulse
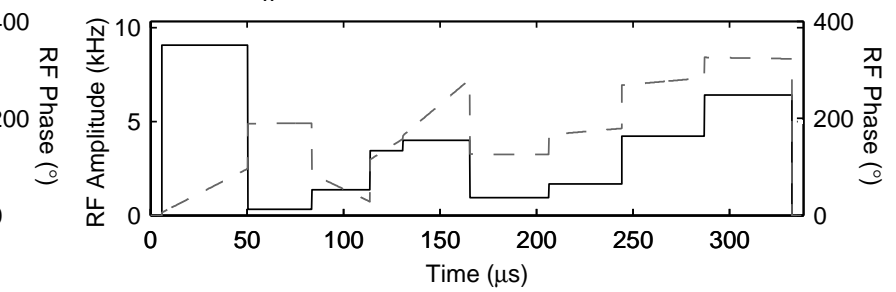

\begin{tabular}{r|c|c|c|c|c|c|c|c|c|} 
Pulse Parameters: & 1 & 2 & 3 & 4 & 5 & 6 & 7 & 8 & 9 \\
\cline { 2 - 10 } Time $(\mu \mathrm{s})$ & 44.3 & 33.3 & 30.1 & 16.9 & 34.7 & 40.9 & 37.9 & 42.7 & 45.4 \\
\cline { 2 - 10 } Power $(\mathrm{kHz})$ & 9.06 & 0.31 & 1.36 & 3.45 & 3.99 & 0.94 & 1.68 & 4.21 & 6.42 \\
\cline { 2 - 10 } Initial Phase $\left(^{\circ}\right)$ & 6 & 9 & 85 & 115 & 164 & 126 & 166 & -92 & -34 \\
\cline { 2 - 10 } Frequency $(\mathrm{kHz})$ & 5.66 & 0.07 & -5.29 & 6.98 & 9.30 & -0.04 & 0.96 & 0.99 & -0.19 \\
\hline
\end{tabular}

FIG. 2. The ideal RF waveform for two example pulses. The solid (dashed) line is the amplitude (phase) of the waveform. Changes in the transmitter frequency (within a single period) were implemented by a discrete linear phase ramp. The sharp discontinuities occur at the transitions between periods. Substantial filtering of these high frequency components (smoothing of the shape) has little effect on the gate fidelity. In order to experimentally implement the pulse, it is converted into a discrete series of amplitudes and phases (order $1 \mathrm{~K}$ long) by sampling the ideal waveform at a constant rate. Details of the pulse parameters (as per Eq. 24) are listed below each waveform. Due to experimental implementation issues, a $6 \mu$ s period with zero RF power (i.e., $H_{e x t}=0$ ) is needed before and after the pulse (see waveform) and must be included to produce the desired propagator. 
a) Three-spin $\pi / 2]_{x}^{3}$ pulse
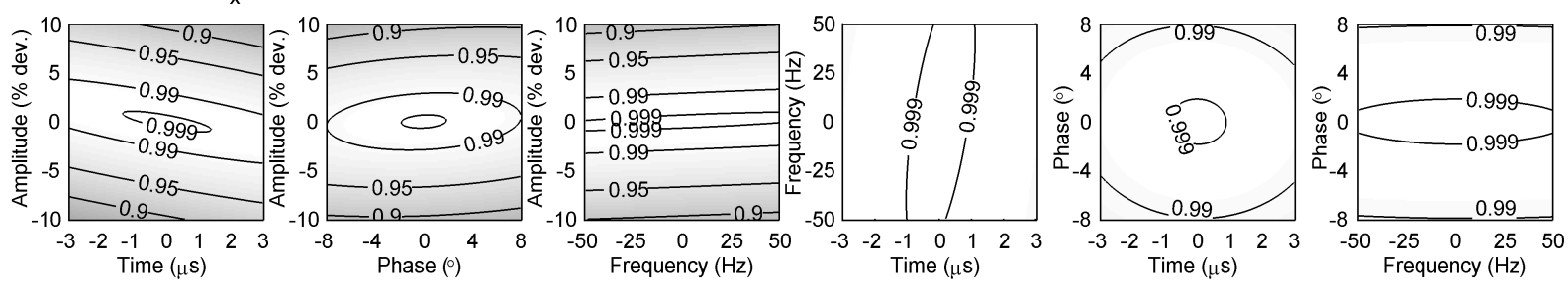

b) Four-spin $\pi]_{x}^{12}$ pulse
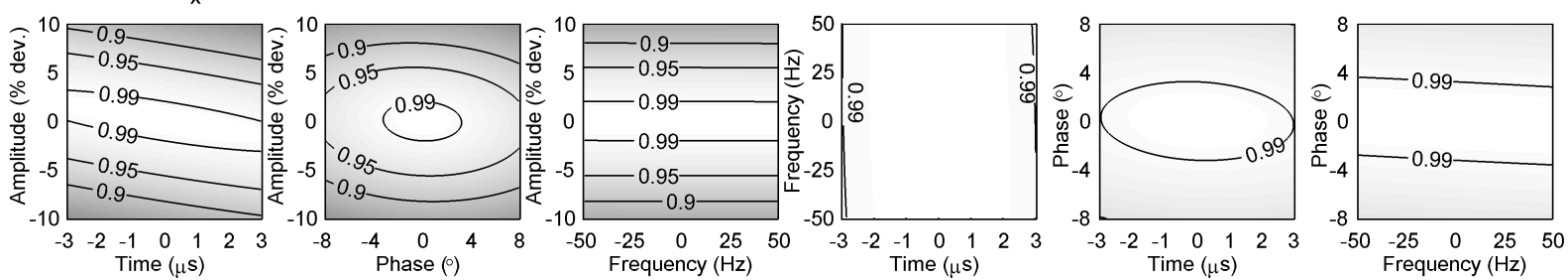

FIG. 3. Contour plots of the gate fidelity as each pair of parameters is varied over typical experimental errors, demonstrating that both the Alanine and Crotonic acid pulses are most sensitive to errors in the RF amplitude.

a) Three-spin $\pi / 2]_{x}^{3}$ pulse

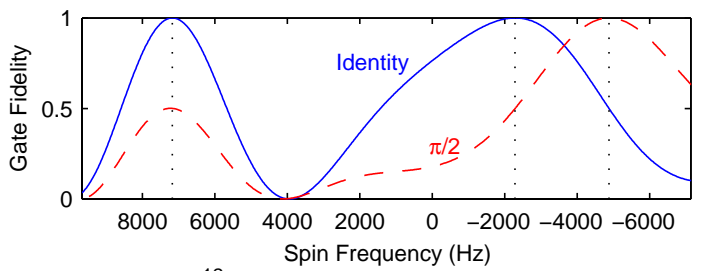

b) Four-spin $\pi]_{x}^{12}$ pulse

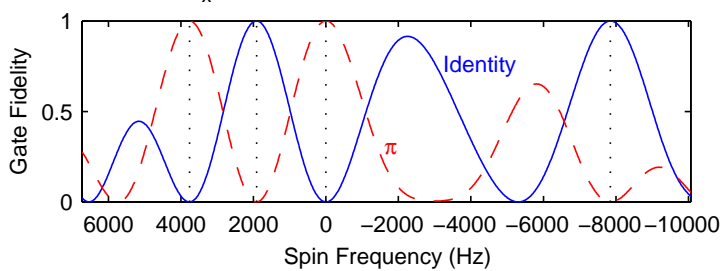

FIG. 4. Gate fidelity of two example pulses as the resonance frequency of a test spin is varied over a range of chemical shifts. The solid (dashed) line is calculated with identity (desired transformation) as the theoretical transformation. The vertical dotted lines denote the actual chemical shifts for each spin. As can be seen, the gate only works when the test spin is at the appropriate resonance frequency. 

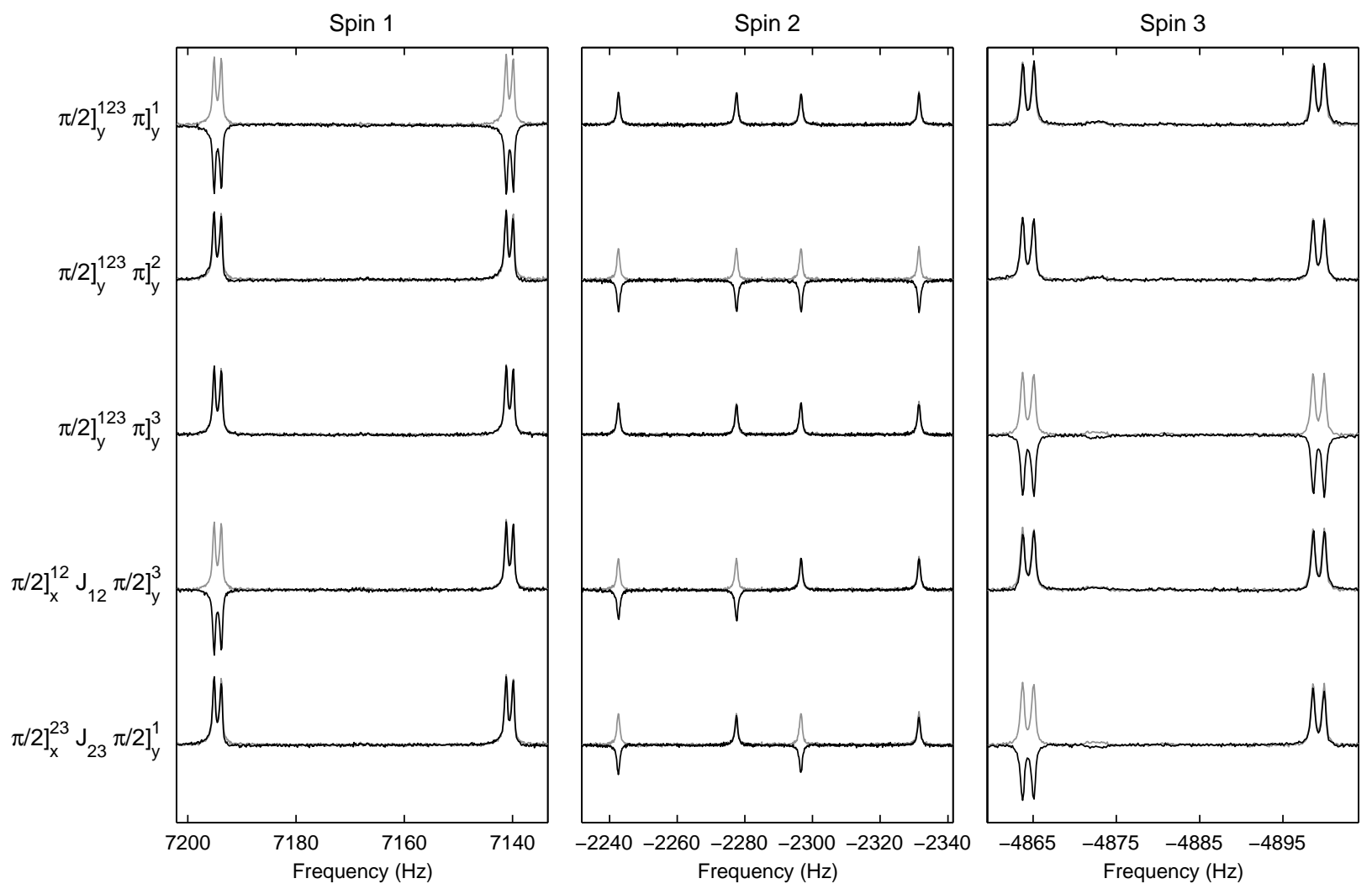

FIG. 5. Spectra resulting from different sequences of pulses applied to the thermal equilibrium density matrix $\rho_{\text {thermal }}=I_{z}^{1}+I_{z}^{2}+I_{z}^{3}$. All sequences are read from left to right. The reference spectra (resulting from a $\frac{\pi}{2}$ pulse applied to all 3 carbons) is also shown (grey) for reference. Although the chemical shift is order $\frac{1}{\tau}$, no significant phase evolution is seen. Selective coupling sequences are also demonstrated. 